\title{
Effect of almonds consumption on anthropometric measurements and blood parameters in overweight and obese females in a weight reduction program
}

\author{
Sabiha İrem Ünal ${ }^{1}$ and Ayla Gulden Pekcan ${ }^{2}$ \\ ${ }^{1}$ Kilometre Nutrition and Diet Counceling Center, Gaziantep, Turkey and \\ ${ }^{2}$ Hasan Kalyoncu University Faculty of Health Sciences Nutrition and Dietetics, Gaziantep, Turkey
}

\section{Abstract}

Nuts are good sources of dietary fiber, magnesium, polyunsaturated fats, vitamin E, and antioxidants, all of which may have health benefits and associated with increased sensations of satiety and could have beneficial effects on weight control. This study aims to determine the effects of a balanced hypocaloric almond (30 g/day) enriched diet (AED) compared to a balanced hypocaloric almond free diet (AFD) on anthropometric measurements and blood parameters. Study was a 3-months, randomized, controlled trial with four serial measurements on 32 overweight and obese (BMI: $\geq 25 \mathrm{~kg} / \mathrm{m}^{2}$ ) women, aged $25-50$ years. The subjects were randomly divided into two groups (16 AED and 16 AFD). Individualized hypocaloric diets of AED considering the energy recommendation, $30 \mathrm{~g} /$ day of almonds was added as a snack. Demographic characteristics, nutritional habits, physical activity levels of subjects were determined by a questionnaire. 24-hour dietary intakes for 3 consecutive days (one day week-end) were recorded at the beginning, 1rd, 2nd, and 3rd months of the study. Anthropometric measurements (height, body weight, waist, hip and neck circumferences) were taken and body composition was determined by Bioelectrical Impedance Analysis (BIA), BMI, waist/hip circumference ratio (WHR) and waist circumference/height ratio (WHtR) were calculated. Fasting blood glucose, insulin level, HbA1c, HOMA-IR, total cholesterol, triglycerides, serum iron, haemoglobin, TSH, CRP were determined prior to and at the end (3rd month) of the study. At the beginning of the study no statistically significant difference was found between anthropometric measurements of the groups $(\mathrm{p}>0.05)$. Body weight, BMI, waist, hip circumferences, WHR, WHtR, percentage of body fat, body fat mass were decreased significantly in almond and control groups compared with the beginning and 1rd, 2nd., 3rd months of the study $(\mathrm{p}=0.001$ ). Significant reductions in HbAlc and LDL-cholesterol levels were determined in almond group $(p<0.05)$. Statistically significant differences were found in insulin, HDL-cholesterol, triglycerides and HOMA-IR levels compared to the prior and end of study in both groups $(\mathrm{p}<0.05)$. Balanced hypocaloric diet caused weight loss and improvements in anthropometric and blood parameters in both groups, but better improvement was determined with almond enriched diet. Almonds as a snack could be recommended as a healthy choice in weight reduction diets. Almonds fiber and protein content may have beneficial effects on weight reduction.

\section{Conflict of Interest}

There is no conflict of interest. 